\title{
Patrón sensibilidad/resistencia en los urocultivos de pacientes con I.T.U. que consultan a urgencias de adultos, Hospital de San José entre enero de 2014 y diciembre de 2014
}

\section{Pattern Sensitivity/Resistance in the Urocultives of Patients with U.T.I. who Consult Adult Urgencies, San Jose Hospital between January 2014 and December 2014}

Diego Armando Riatiga Ibañez ${ }^{1}$ Adriana Jiménez Rojas ${ }^{1}$ Luis Eduardo Salgado Sánchez ${ }^{2}$ Fernando Enrique Gaona Fernández ${ }^{2}$ Yesica Yasmín Quiroz Madarriaga ${ }^{1}$

1 Departamento de Urología, Fundación Universitaria de Ciencias de la
Salud, Hospital de San José de Bogotá, Bogotá, Colombia
${ }^{2}$ Departamento de Urología, Hospital de San José, Bogotá, Colombia

Urol Colomb 2019;28:296-302
Address for correspondence Yesica Quiroz Madarriaga, MD, Urologist, Departamento de Urología, Hospital de San José, Hospital san José Calle 10 \#18-75, Bogotá, Colombia (e-mail: yeyaquimapa@hotmail.com).

\section{Resumen}

Palabras Clave

- infección del tracto urinario (ITU)

- síntomas obstructivos urinarios bajos (SOUB)

- betalactamasas de espectro extendido (BLEA)

- bacterias productoras de carbapenemasas (AMPc, KPC)
Introducción: La infección del tracto urinario (ITU) es un motivo de consulta frecuente en urgencias, siendo necesario el tratamiento antibiótico empírico antes de obtener un urocultivo.

Objetivo Determinar el patrón de sensibilidad/resistencia de los gérmenes aislados en los urocultivos de pacientes que ingresan por urgencias de adultos con sintomatología sugestiva de ITU.

Métodos Se realizó un estudio observacional, descriptivo de corte transversal, recolectando los datos de los urocultivos de pacientes con diagnóstico de ITU que asistieron por urgencias adultos, y cumplieron los criterios de inclusión en el año 2014.

Resultados Se analizaron 133 pacientes (133 urocultivos), 50,4\% mujeres, edad promedio de 64,1 años (DE 19,1). Se encontró una frecuencia mayor de ITU alta (54,9\%), e ITU no complicada (54,1\%), la mayoría de origen en la comunidad (95,5\%). El germen más frecuentemente aislado fue $E$. coli $(56,4 \%)$, seguido por Klebsiella oxytoca (7,1\%). La resistencia de $E$. coli fue del 55,7\% para ampicilina sulbactam, 58,2\% para nitrofurantoina, $40 \%$ para quinolonas y $32 \%$ para cefazolina. En pacientes con factores de riesgo urológico se encontró una resistencia entre el $40 \%$ al $50 \%$. Se evidenció patrón BLEA (12,9\%), AMPc (20\%) y KPC (1,4\%).

Conclusión Los uropatógenos han aumentado de forma significativa su resistencia a las diferentes familias de antibióticos, entre ellas las cefalosporinas y las quinolonas, confirmando que medicamentos como ampicilina sulbactam, nitrofurantoina, y ciprofloxacina no están indicados en el manejo empírico de ITU en nuestra institución, especialmente al presentar factores de riesgo urológicos. received

February 8, 2018

accepted

July 13, 2018
DOI https://doi.org/

$10.1055 / \mathrm{s}-0038-1668514$.

ISSN 0120-789X.

e ISSN 2027-0119.
Copyright (c) 2019, Sociedad Colombiana License terms de Urología. Publicado por Thieme Revinter Publicações Ltda., Rio de Janeiro, Brazil. Todos los derechos reservados.

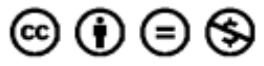


Abstract

\section{Keywords}

- urinary tract infection (UTI)

- low urinary tract symptoms (LUTS)

- extended spectrum $\beta$ lactamases (BLEA)

- carbapenemaseproducing bacteria (cAMP, KPC)
Introduction Urinary tract infection (UTI) is a frequent cause for assistance to the emergency services, in most cases is necessary initiate empirical antimicrobial management while obtaining the result of the uroculture. Therefore, it is vital to know the pattern of sensitivity/resistance of the most frequent uropathogens and thus offer the best initial treatment.

Objective To determine the pattern of sensitivity / resistance of isolated germs in the urine cultures of adults patients admitted to the emergency room with UTI suggestive symptoms.

Methods An observational, descriptive, cross-sectional study was conducted, collecting urine culture data from patients diagnosed with UTI who were attended in adult emergencies in 2014.

Results A total of 133 patients were analyzed (133 urine cultures), 50.4\% were women, with average age of 64.1 years (SD 19.1). Higher frequency of high urinary tract infection was found (54.9\%), and uncomplicated UTI (54.1\%), most UTI originated in the community (95.5\%). The most frequently isolated germ was E. coli (56.4\%), followed by Klebsiella oxytoca (7.1\%). The resistance of $E$. coli was $55.7 \%$ for ampicillin sulbactam, $58.2 \%$ for nitrofurantoin, $40 \%$ for quinolones and $32 \%$ for cefazolin. In patients with urological risk factors, resistance was found between $40 \%$ and $50 \%$. BLEA pattern (12.9\%), cAMP (20\%) and KPC (1.4\%) were evidenced.

Conclusion Uropathogens have significantly increased their resistance to different families of antibiotics, including cephalosporins and quinolones, confirming that drugs such as ampicillin sulbactam, nitrofurantoin, and ciprofloxacin are not indicated in the empirical management of UTI in our institution, especially with the presence of urological risk factors.

\section{Introducción}

La infección del tracto urinario (ITU) está definida como la respuesta inflamatoria del urotelio secundaria a una invasión bacteriana que suele asociarse con bacteriuria y piuria. Esa patología es frecuente afectando a hombres y mujeres de todas las edades con presentación y secuelas variables causando alta morbilidad y una tasa de mortalidad significativa; causa 7 millones de consultas médicas por año en EEUU. ${ }^{1}$

Se han descrito tres vías como patogenia de la infección, siendo la más frecuente la vía ascendente, seguida de la vía hemática y linfática; se calcula que aproximadamente un $10 \%$ de la población mundial presenta una ITU durante su vida. ${ }^{2}$ Escherichia coli ocupa el primer lugar como agente causal de ITU no complicada siendo responsable del $75 \%$ al $95 \%$ de los casos, seguida de enterobacterias como Proteus mirabilis y Klebsiella pneumoniae. Así mismo, en el caso de la ITU no complicada se encuentran microorganismos como Pseudomona, Serratia y Providencia entre otros, ${ }^{3-5}$ donde Pseudomona ha mostrado tener menor sensibilidad a antibióticos comparada con otros microorganismos. ${ }^{6}$

La elección de manejo antibiótico empírico se ha convertido en un reto dada la creciente resistencia antibiótica a cefalosporinas, trimetoprim sulfametoxazol y quinolonas, y la importancia de esos en el efecto que tiene en morbilidad, mortalidad y costos al sistema de salud. ${ }^{7}$ Así mismo, la colonización previa del tracto urinario, fenómeno presente tanto en medio hospitalario como la comunidad, es un factor importante al momento de decidir el antibiótico que será instaurado en cada paciente. ${ }^{8}$ Debe tenerse en cuenta el microorganismo sospechado y el patrón de resistencia con base en el escenario clínico y los patrones de resistencia locales. ${ }^{7-9}$

Según el departamento de estadística del Hospital de San José, Bogotá - Colombia las infecciones urinarias son un motivo frecuente de consulta a nuestro servicio de urgencias. Para el año 2012, la infección de vías urinarias fue la tercera causa dentro de las patologías más frecuentes en el servicio de urgencias, con un número estimado de 1248 pacientes. ${ }^{10}$ Es por ello que este estudio pretende establecer el patrón de sensibilidad/resistencia que tienen las bacterias que más frecuentemente se aíslan en los urocultivos de pacientes que consultan a urgencias del Hospital de San José y comparar esos resultados con los descritos en nuestra publicación en $2009 .^{11}$

\section{Métodos}

Se realizó un estudio observacional, descriptivo de tipo corte transversal, revisando de manera retrospectiva los registros médicos y urocultivos de pacientes que ingresaron por el servicio de urgencias adultos del Hospital de San José con sintomatología urinaria y diagnóstico CIE-10 (N390) durante el período de 12 meses comprendido desde el 1 enero hasta el 
31 de diciembre del año 2014; esos registros se obtuvieron de la base de datos del laboratorio del hospital.

Se determinaron como criterios de exclusión pacientes con hospitalización reciente extra institucional (últimas 2 semanas), antecedente de biopsia transrectal de próstata y antecedente quirúrgico extrainstitucional con instrumentación de vía urinaria en las últimas 2 semanas previas al diagnóstico de infección del tracto urinario. Se definieron las variables a estudio: urocultivo positivo como el aislamiento de un germen uropatógeno con más de 100.000 unidades formadoras de colonias ${ }^{1}$; infección del tracto urinario como la respuesta inflamatoria del urotelio secundaria a una invasión bacteriana que suele asociarse con bacteriuria y piuria'; infección urinaria complicada como la que se presenta con una alteración anatómica o funcional del tracto urinario, ${ }^{1}$ manipulación reciente extra institucional del tracto urinario (últimas 2 semanas), o uso de dispositivos dentro de la vía urinaria; Infección urinaria no complicada como la que se presenta sin alteración anatómica o funcional del tracto urinario, definida por ecografía renal y de vías urinarias; infección urinaria alta como la presencia de compromiso sistémico dado por fiebre, malestar general, leucocitosis ${ }^{1}$; e infección urinaria baja la cual se presenta con síntomas como disuria, polaquiuria, tenesmo, sin compromiso sistémico ${ }^{1,3}$

Así mismo se definieron BLEA, AMPc y KPC como: germen inductor de betalactamasa de espectro extendido, germen que expresa resistencia a betalactamasas tipo AmpC y germen inductor de carbapenemasas, respectivamente. ${ }^{12}$

Se evaluaron las historias clínicas electrónicas de esos pacientes con el fin de establecer el patrón de sensibilidad / resistencia reportados en los urocultivos los cuales fueron tomados bajo el protocolo instaurado por el comité de infecciones de esta institución, la cual se encuentra acreditada por ICONTEC. Su análisis fue realizado mediante un panel automatizado por MicroScan Walk Away 96 Plus®.

La base de datos se construyó en Excel 2007 y el análisis estadístico de la información se realizó en Stata 13® (Data
Analysis and Statistical Software); se utilizó estadística descriptiva, las variables cualitativas se reportaron con frecuencias absolutas y relativas; y las variables cuantitativas se reportan con medidas de tendencia central y dispersión. La unidad de análisis fueron los reportes de urocultivos.

El protocolo fue aprobado por el comité de investigaciones de la facultad de medicina y el comité de ética de investigación con seres humanos del Hospital San José.

\section{Resultados}

Se revisaron 260 historias clínicas de pacientes a quienes se les realizó urocultivo en el servicio de urgencias adultos del Hospital San José durante el periodo de estudio, de los cuales 94 tuvieron un resultado negativo y 166 fueron positivos, se aplicaron los criterios de exclusión, descartando 33 urocultivos (-Fig. 1).

Se incluyeron 133 urocultivos que corresponden a 133 pacientes, analizando los datos según el género, en un 50,4\% femenino y 49,6\% masculino, la edad promedio fue de 64,1 años en un rango de 19 a 97 años. Al revisar las historias clínicas, en busca de antecedentes relevantes (patologías urológicas e instrumentación de vía urinaria) en la población de estudio, 49,6\% no presentó ningún antecedente y 50,4\% presentó al menos un antecedente, siendo los más frecuentes: la manipulación de la vía urinaria (27,8\%), seguido de presencia de síntomas obstructivos urinarios bajos (26,3\%) y la presencia de dispositivo en vía urinaria (24\%), (- Tabla 1).

Al evaluar el tipo de infección, se encontró una frecuencia mayor de ITU alta (54,9\%), al igual que la presencia de ITU no complicada $(54,1 \%)$, siendo la mayoría de origen en la comunidad (95,5\%). ( - Tabla 1).

De los 133 pacientes con urocultivos positivos, se aislaron 140 gérmenes siendo 5 urocultivos positivos para 2 gérmenes y uno positivo para 3 gérmenes uropatógenos. El germen más frecuentemente aislado fue Escherichia coli en 56,4\%, seguido por Klebsiella oxytoca (7,1\%), Citrobacter freundii (4,3\%),

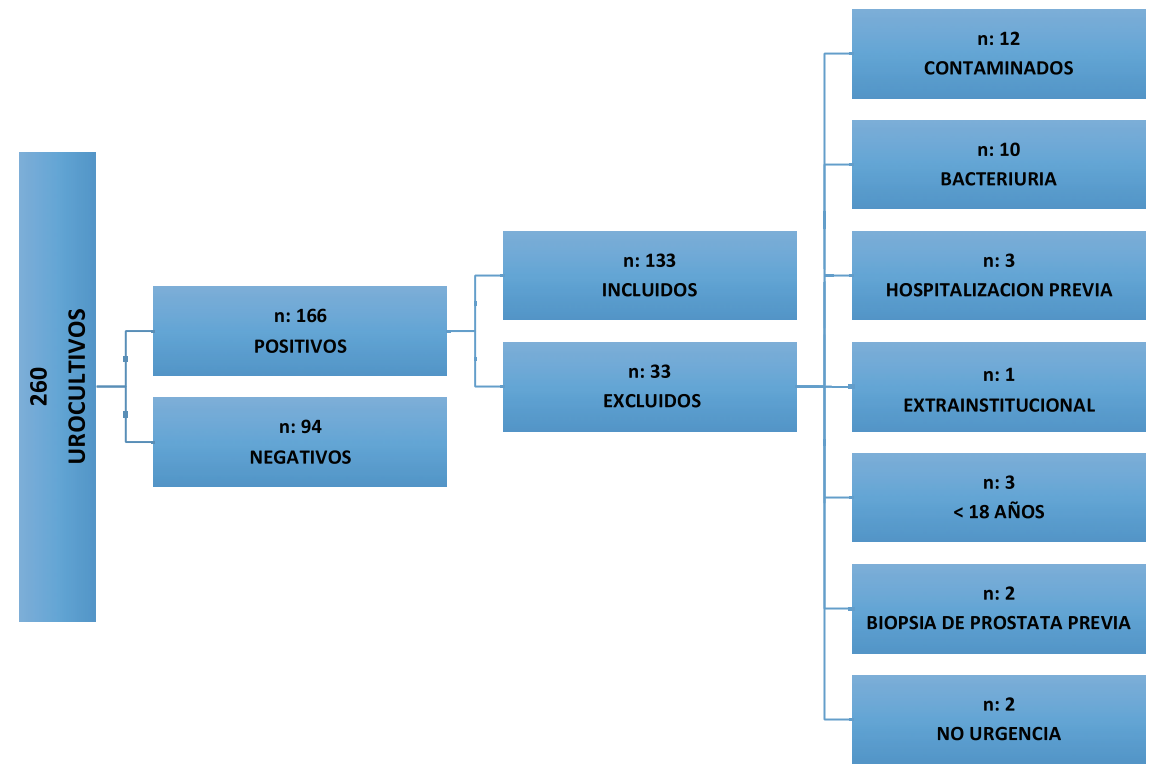

Fig. 1 Urocultivos seleccionados para el estudio. 
Tabla 1 Características de la población de pacientes adultos con IVU

\begin{tabular}{|l|l|}
\hline Características & $\mathbf{N}=133$ \\
\hline Edad, años. Promedio (DE) & $64,1(19,1)$ \\
\hline Mínima - Máxima & 19 - 97 \\
\hline Sexo masculino, n (\%) & $66(49,6)$ \\
\hline Masculino & $67(50,4)$ \\
\hline Femenino & $8(6)$ \\
\hline Antecedentes, n (\%) & $3(2,3)$ \\
\hline Vejiga neurógena & $35(26,3)$ \\
\hline Reflujo vesico-ureteral & $32(24)$ \\
\hline S.O.U.B ${ }^{a}$ & $10(7,5)$ \\
\hline Dispositivo en vía urinaria & $37(27,8)$ \\
\hline Litiasis & $60(45,1)$ \\
\hline Manipulación de vía urinariab & $73(54,9)$ \\
\hline Tipo de Infección, n (\%) & $72(54,1)$ \\
\hline Baja & $61(45,9)$ \\
\hline Alta & $127(95,5)$ \\
\hline No Complicada & \multicolumn{2}{|l}{} \\
\hline Complicada & \\
\hline Origen, n (\%) & \\
\hline Nosocomial & \\
\hline Comunidad & \\
\hline
\end{tabular}

áíntomas obstructivos del tracto urinario bajo.

${ }^{\mathrm{b}}$ Antecedente de manipulación del tracto urinario, incluidos procedimientos quirúrgicos.

Enterococcus faecalis $(4,3 \%)$ y el resto de microorganismos aislados se encontraron por debajo del 4\% (Pseudomona auriginosa, Proteus mirabilis y vulgaris, Enterobacter aerogenes y cloacae, Providencia rettgeri, Serratia marcescens, Klebsiella pneumoniae, Morganella morgani, Achromobacter xylosoxidans, Citrobacter koseri, Enterococcus faecium, Kluyvera ascorbada, Sthaphylococcus aureus y sciuri) (-Fig. 2).

La incidencia de $E$. coli con el fenotipo de producción de betalactamasas de espectro ampliado (BLEA) fue alta, según
Tabla 2 Sensibilidad - Resistencia de los microorganismos más frecuentemente aislados pacientes adultos con ITU que ingresan a urgencias. Hospital de San José 2014

\begin{tabular}{|l|l|l|l|l|}
\hline & $\begin{array}{l}\text { E. coli } \\
\mathbf{n : 7 9}\end{array}$ & $\begin{array}{l}\text { K. oxytoca } \\
\mathbf{n}: 10\end{array}$ & C. freundiin:6 & $\begin{array}{l}\text { E. faecalis } \\
\mathbf{n}: 6\end{array}$ \\
\cline { 2 - 5 } & $\begin{array}{l}\mathbf{S}=\mathbf{n} \\
(\%)\end{array}$ & $\mathbf{S}=\mathbf{n}(\%)$ & $\mathbf{S}=\mathbf{n}(\%)$ & $\begin{array}{l}\mathbf{S}=\mathbf{n} \\
(\%)\end{array}$ \\
\hline Ampicilina & $27(34.2)$ & $0(0)$ & $1(17)$ & $6(100)$ \\
\hline Amoxicilina & $28(35.4)$ & $1(10)$ & $1(17)$ & $6(100)$ \\
\hline Ciprofloxacina & $48(60.8)$ & $9(90)$ & $5(83)$ & $5(83)$ \\
\hline Norfloxacina & $47(59.5)$ & $9(90)$ & $5(83)$ & $5(83)$ \\
\hline Gentamicina & $57(72.2)$ & $9(90)$ & $4(67)$ & $5(83)$ \\
\hline Amikacina & $75(94.9)$ & $10(100)$ & $6(100)$ & $6(100)$ \\
\hline Nitrofurantoína & $33(41.8)$ & $6(60)$ & $1(17)$ & $2(33)$ \\
\hline Ceftriaxona & $61(77.2)$ & $8(80)$ & $3(50)$ & $6(100)$ \\
\hline Imipenem & $79(100)$ & $10(100)$ & $6(100)$ & $6(100)$ \\
\hline Ertapenem & $79(100)$ & $10(100)$ & $6(100)$ & $6(100)$ \\
\hline $\begin{array}{l}\text { Piperacilina/ } \\
\text { Tazobactam }\end{array}$ & $72(91.1)$ & $9(90)$ & $5(83)$ & $6(100)$ \\
\hline $\begin{array}{l}\text { Ampicilina/ } \\
\text { Sulbactam }\end{array}$ & $35(44.3)$ & $5(50)$ & $1(17)$ & $2(33)$ \\
\hline Cefazolina & $53(67.1)$ & $8(80)$ & $2(33)$ & $4(67)$ \\
\hline
\end{tabular}

se deduce de la resistencia a ampicilina/sulbactam (55,7\%) y cefazolina (32,9\%). El porcentaje de bacterias productoras de BLEA fue de $12,8 \%$. La sensibilidad a la nitrofurantoina para todos los microorganismos aislados fue de $24 \%$. No se detectó la presencia de E. coli productora de carbapenemasa y se identificó tipificación de dos Klebsiellas pneumoniae con fenotipo de KPC. El $17 \%$ de los microorganismos presentaron un patrón AMPc inducible o reprimido.

En la - Tabla 2 se presenta la sensibilidad específica a cada antibiótico en los microorganismos aislados con mayor frecuencia; no se reportaron los datos de sensibilidad / resistencia de 39 microorganismos por su baja frecuencia en aislamiento.

Dentro de los pacientes con aislamiento de E. coli en los urocultivos, los factores de riesgo urológicos más frecuentemente presentados fueron manipulación de la vía urinaria $(19,4 \%)$, síntomas obstructivos urinarios bajos

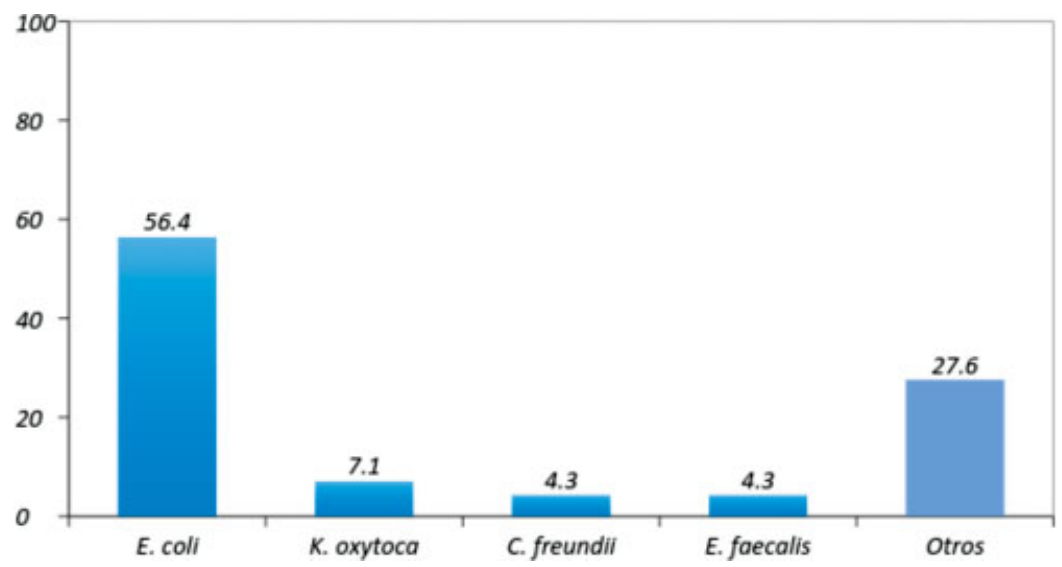

Fig. 2 Microorganismos aislados en urocultivos revisados 
Tabla 3 Sensibilidad - Resistencia de E. coli con factores de riesgo urológicos (N: 84)

\begin{tabular}{|l|l|l|l|l|}
\hline & $\begin{array}{l}\text { SOUB } \\
\text { N 12 }(\%)\end{array}$ & $\begin{array}{l}\text { Dispositivo vía Urinaria } \\
\text { N 10 (\%) }\end{array}$ & $\begin{array}{l}\text { Manipulación } \\
\text { vía Urinaria } \\
\text { N 15 (\%) }\end{array}$ & $\begin{array}{l}\text { Sin Factor de Riesgo } \\
\text { N 47 (\%) }\end{array}$ \\
\hline Ampicilina & $4(33,3)$ & $2(20)$ & $2(13,3)$ & $18(38,2)$ \\
\hline Amoxicilina & $4(33,3)$ & $2(20)$ & $2(13,3)$ & $18(38,2)$ \\
\hline Ciprofloxacina & $3(25)$ & $3(30)$ & $6(40)$ & $33(70,2)$ \\
\hline Norfloxacina & $3(25)$ & $3(30)$ & $6(40)$ & $32(68)$ \\
\hline Gentamicina & $7(58,3)$ & $5(50)$ & $8(53,3)$ & $37(78,7)$ \\
\hline Amikacina & $12(100)$ & $8(80)$ & $14(93,3)$ & $46(99,9)$ \\
\hline Nitrofurantoína & $3(25)$ & $2(20)$ & $6(40)$ & $21(44,6)$ \\
\hline Ceftriaxona & $12(100)$ & $4(40)$ & $7(46,6)$ & $36(76,5)$ \\
\hline Imipenem & $12(100)$ & $10(100)$ & $15(100)$ & $47(100)$ \\
\hline Ertapenem & $12(100)$ & $10(100)$ & $13(86,6)$ & $47(100)$ \\
\hline Piperacilina/Tazobactam & $10(83,3)$ & $9(90)$ & $3(20)$ & $95(95,7)$ \\
\hline Ampicilina/Sulbactam & $4(33,3)$ & $3(30)$ & $9(60)$ & $23(48,9)$ \\
\hline Cefazolina & $7(58,3)$ & $5(50)$ & $31(65,9)$ \\
\hline
\end{tabular}

${ }^{a}$ Síntomas obstructivos del tracto urinario bajo.

$(15,5 \%)$ y dispositivo en la vía urinaria (12,9\%), encontrando una sensibilidad a cefalosporinas de primera generación entre el $50 \%$ y $60 \%$, la cual al ser comparada con la sensibilidad en los pacientes sin ningún factor de riesgo urológico fue menor.

En la - Tabla 3 se presenta la sensibilidad específica a cada antibiótico en los factores de riesgo urológicos.

\section{Discusión}

En Latinoamérica, los estudios diseñados para la evaluación de patrones de sensibilidad/resistencia de los agentes etiológicos de ITU en servicios de urgencias son limitados. En Colombia, diferentes redes de vigilancia a la resistencia bacteriana han establecido los patrones de los aislamientos por institución, más no por servicio de atención (GREBO - Grupo para el control de la resistencia bacteriana en Bogotá). ${ }^{13}$ El Servicio de Urología del Hospital de San José constituye un centro de remisión de patología urológica complicada para el centro y sur de Bogotá, eso determina que se atienda un alto porcentaje de pacientes con antecedentes de síntomas obstructivos urinarios bajos e instrumentación de la vía urinaria. Se ha establecido que las anteriores comorbilidades predisponen a adquirir ITU a repetición e inducen la selección de microorganismos con patrones de resistencia adquiridos como los identificados en este estudio. $^{10}$

Como se evidenció previamente en los resultados, E. coli continua siendo el germen más frecuentemente aislado en los pacientes con infección del tracto urinario, lo cual es acorde a lo reportado en la literatura. En Uruguay Palacio y colaboradores (1998), buscaron identificar la resistencia de los antibióticos de patógenos bacterianos aislados de infección urinaria alta. En tres hospitales y durante 2 años se realizó la identificación de cepas invasivas aisladas de pacientes hospitalizados, identificando 299 cepas de 29 especies diferentes, entre las cuales predomino E. Coli con un $85 \%$. Sin embargo, la incidencia de los demás uropatógenos fue diferente a lo reportado en este estudio dado que su población es diferente a la nuestra. ${ }^{14}$

No es posible establecer una comparación entre infecciones nosocomiales y adquiridas en la comunidad, debido al bajo número de infecciones nosocomiales que se obtuvieron en esta revisión, tan solo 6, de las cuales 3 fueron producidas por $E$. coli, una de ellas con patrón de resistencia BLEA, una S. marcescens con patrón de resistencia AMPc y P. rettgeri KPC. Generalmente, en pacientes con infección intrahospitalaria, la frecuencia de Escherichia coli disminuye, mientras que aumenta la especie de Klebsiella, Enterobacter, Citrobacter, Serratia, Pseudomona aeruginosa, Providencia spp y Enterococcus spp. ${ }^{15}$ Ese hallazgo se confirma en un estudio realizado en Cartagena en el 2010, sobre 455 urocultivos positivos realizados a 137 pacientes hospitalizados y 318 ambulatorios, en donde se encontró que el $86,9 \%$ pertenecía a la familia Enterobacteriaceae. ${ }^{16}$

En el presente estudio se encuentra una alta resistencia de la E. coli a cefalosporinas de primera y tercera generación (34 y $24 \%$ respectivamente), al igual que a betalactamicos asociados a inhibidores de betalactamasas (51\%), esos hallazgos corresponden a paciente sin factores de riesgo, en pacientes con factores de riesgo la resistencia a ampicilina sulbactam asciende al 66,7\%. El estudio de Gordon y col., (2013), muestra que el patrón de resistencia de E. coli, K. pneumoniae y P. mirabilis frente a betalactámicos con inhibidor de betalactamasa (ampicilina/sulbactam y amoxicilina/clavulonato) estuvo entre 50 y $80 \%$ y la resistencia de E. coli a cefalosporinas de $2^{\underline{a}}$ y $3^{\text {a }}$ generación fue de 63 y 36,4\% respectivamente. ${ }^{16}$ También Chavez y col., (2010) reportaron que la E. Coli fue la bacteria más frecuentemente aislada en pacientes con infección del tracto urinario no complicada tanto hospitalizados como ambulatorios, encontrando una alta resistencia de este microorganismo a betalactámicos con inhibidor de betalactamasa (ampicilina/sulbactam y amoxicilina/ 
clavulonato), pero una alta sensibilidad a cefalosporinas de $2^{\text {a }}$ generación $(90,9-81 \%){ }^{17}$

Machado y col. (2012) publicaron un estudio sobre la evaluación de sensibilidad antibiótica en urocultivos de pacientes en primer nivel de atención en salud en la ciudad de Pereira. Fueron tomados 1058 urocultivos positivos, de los cuales no se discriminaron entre hospitalarios y ambulatorios. El uropatógeno más frecuentemente aislado fue E. coli $(67,2 \%)$, seguido de Klebsiella spp (19,2\%) y Enterococcus spp. E. coli mostró alta resistencia a la ampicilina (54,7\%), alta resistencia a Piperacilina Tazobactam (60\%) y resistencia del 28,1\% a Ciprofloxacina, lo cual no se relaciona con nuestros resultados, debido a que a pesar de encontrar una resistencia superior al 60\% para ampicilina, la sensibilidad a piperacilina tazobactam en nuestro estudio fue mucho mayor (95,7\%). (- Tabla 2). ${ }^{18}$

En Colombia, Jiménez y col. (2014), encontraron que la insuficiencia renal, la cirugía urológica, el antecedente de uso de antibiótico en los 3 meses anteriores, el origen nosocomial de la infección y la hospitalización previa constituyen factores de riesgo para el aislamiento de una enterobacteria productora de BLEA. A pesar de que en el hospital está restringido el uso de quinolonas desde hace dos décadas, se encontró una alta resistencia a las mismas (28\%), así como enterobacterias de BLEA (19\%) y resistencia a nitrofurantoina (53\%). La resistencia a ese tipo de antibióticos, determina que no puedan ser incluidos en el protocolo de manejo empírico de la ITU en pacientes con patología urológica. La selección del antibiótico empírico en ese tipo de pacientes, dependerá de establecer la epidemiologia local y la evaluación de factores de riesgo específicos. ${ }^{10}$

Otro estudio colombiano que buscó establecer los patrones de sensibilidad y resistencia en urocultivos fue el realizado por Gomez y cols., en el 2009, en donde revisaron 2.312 urocultivos de una base de datos que incluía todos los aislamientos de una institución en la ciudad de Bogotá (1618 extra-hospitalarios y 694 intra-hospitalarios), siendo $E$. coli el germen más frecuentemente aislado con un $62,58 \%$ de los casos, presentando resistencia a trimetropim-sulfametoxazol en el $43,4 \%$ de los aislamientos, a las quinolonas como la ciprofloxacina 31,4\%, ampicilina $51,9 \%$ y ampicilinasulbactam $32,2 \%$. Sin embargo, reportaron sensibilidades muy altas para nitrofurantoína 98,3\%, cefalosporinas de primera $91,24 \%$, segunda 92,5\% y tercera generación 97,9\%. Dada la diferencia a lo encontrado en el presente estudio, podríamos asumir que esa sensibilidad se vio influenciada por la inclusión de pacientes ambulatorios, debido a que son los pacientes con menos factores de riesgo para presentar $\mathrm{u}$ incremento en la resistencia bacteriana. ${ }^{19}$

En el Hospital de San José (Bogotá - Colombia) para el año 2009 fue realizado un estudio con el objetivo de identificar el patrón de sensibilidad/resistencia antimicrobiana en ITU de pacientes que acudieron al servicio de urgencias y fueron atendidos exclusivamente por el servicio de urología. Se analizaron 92 urocultivos encontrando que $E$. coli continuaba siendo el germen más frecuente dentro de los cuadros de ITU, con una resistencia amikacina y nitrofurantoina del $8,5 \%$, cefazolina del $13,6 \%$ y piperazilina tazobactam del 10,2\%, mientras que presentaba altos porcentajes de resistencia para ampicilina 57,6\%, ampicilina-sulbactam 32,2\%, norfloxacina 23,7\%, ciprofloxacina $22 \%$ y gentamicina $13,8 \%{ }^{11}$ Lo anterior, al ser comparado con los resultados del presente estudio (mismos criterios de inclusión y exclusión), la resistencia de las cepas de E. Coli registradas aumento para cefazolina a $32,9 \%$ y para nitrofurantoina a $59,8 \%$; mientras la resistencia para ampicilina aumento a $65,8 \%$, ampicilina sulbactam a $55,7 \%$, norfloxacina 40,5\%, ciprofloxacina $39,2 \%$ y gentamicina $27,8 \%$.

En este estudio se puede citar que los pacientes se seleccionaron exclusivamente del servicio de urgencias y se analizaron los patrones de sensibilidad discriminados por factores de riesgo urológicos lo cual permite establecer un patrón epidemiológico como fundamento para la construcción de protocolos. No se realizó la búsqueda de bacterias atípicas como Mycoplasma sp., Chlamydia sp., o virus. En nuestro estudio no fue posible la estandarización de criterios clínicos para la selección de pacientes para toma de urocultivo por parte de todo el personal del servicio de urgencias del Hospital de San José, lo cual pudo afectar nuestra población de estudio al excluir pacientes con criterios de ITU para toma de urocultivos. Por tratarse de una institución de alto nivel de complejidad, los pacientes que cursan con infecciones urinarias no complicadas no suelen consultar a nuestra institución, sino a centros de mi primer nivel, por lo que nuestros resultados no son extrapolables a ese tipo de población.

\section{Conclusión}

Identificar el tipo de microorganismos y el patrón de sensibilidad antimicrobiana asociados a ITU en un servicio de urgencias, permite establecer los protocolos de manejo empírico de esta patología. En este estudio se confirma que antibióticos como ampicilina sulbactam, nitrofurantoína y ciprofloxacina no deben ser empleados en el manejo empírico de ITU en este tipo de hospitales, especialmente en pacientes con factores de riesgo urológicos debido a la elevada resistencia de los uropatógenos a esos antimicrobianos. También se evidencia un aumento en la resistencia antimicrobiana para antibióticos como cefazolina y dado su frecuente uso en el manejo inicial de los pacientes con ITU que ingresan al servicio de urgencias, se podrían establecer líneas diferentes de tratamiento inicial para ITU en pacientes con factores de riesgo urológico (SOUB, dispositivo en vía urinaria, manipulación de vía urinaria), como piperacilina/tazobactam y carbapenémicos.

\section{Agradecimientos}

Se agradece al Hospital de San José, a la división de investigaciones, al laboratorio clínico y al departamento de microbiología por su colaboración y paciencia durante la realización de este proyecto.

Declaración De Financiación Del Proyecto

Proyecto financiado por el servicio de Urología. Hospital de San José y División de Investigaciones Fundación Universitaria de Ciencias de la Salud. 
Consideraciones Éticas

El presente estudio tiene en cuenta para su realización los parámetros expuestos en el Código de Núremberg, la Declaración de Helsinki, el informe Belmont, las pautas CIOMS y la resolución $\mathrm{N}^{\circ} 008430$ de 1993 del Ministerio de Salud de Colombia se considera una investigación sin riesgo. El protocolo se presentará ante el comité de investigaciones de la facultad de medicina y el comité de ética de investigación con seres humanos.

Los investigadores declaran que no tienen ningún conflicto de interés. Se mantendrá oculta la identidad de los pacientes para el análisis de las bases de datos.

\section{Conflicto de intereses}

Los autores declaran no tener ningún conflicto de intereses.

\section{Referencias}

1 Wein A, Kavoussi L, Novick A, Partin A. Campbell Walsh Urology. 10th ed 2012

2 Sultana S, Khatun M, Khoybar M, Mawla N. Pattern of antibiotic sensivity of bacteria causing urinary tract infection in a private Medical College Hospital in Dhaka. Bangladesh J Med Sci 2015; 14(01):70-74

3 Hooton T, Calderwood S, Bloom A. Acute complicated cystitis and pyelonephritis http://www.uptodate.com/store2015 [cited 2015 Abril 15].

4 Saha S, Nayak S, Bhattacharyya I, et al. Understanding the patterns of antibiotic susceptibility of bacteria causing urinary tract infection in West Bengal, India. Front Microbiol 2014;5:463

5 Niranjan V, Malini A. Antimicrobial resistance pattern in Escherichia coli causing urinary tract infection among inpatients. Indian J Med Res 2014;139(06):945-948

6 Yoon BI, Kim HS, Kim SD, et al. Changes in bacterial species and antibiotic sensitivity in intensive care unit: acquired urinary tract infection during 10 years interval (2001-2011). Urol J 2014;11 (02):1478-1484

7 MacFadden DR, Ridgway JP, Robicsek A, Elligsen M, Daneman N. Predictive utility of prior positive urine cultures. Clin Infect Dis 2014;59(09):1265-1271
8 Suárez B, Milián Y, Espinoza F. Susceptibilidad antimicrobiana y mecanismos de resistencia de Escherichia coli aisladas a partir de urocultivos en un hospital de tercer nivel. Rev Cubana Med 2014; 53(01):3-13

9 Oyekale T, Fadare O, Busari A, Adeyemo T. Antibiotic resitance pattern of bactrial isolates from cases of urinary tract infections among hospitalied and out-patients at a tertiary health facility in South Western Nigeria. Ann Trop Med Public Health 2014;7(02): 130-135

10 Jiménez A, Alvarado A, Gómez F, Carrero G, Fajardo C. Factores de riesgo asociados al aislamiento de Escherichia coli o Klebsiella pneumoniae productoras de betalactamasas de espectro extendido en un hospital de cuarto nivel en Colombia. Biomedica 2014;34(01, Suppl 1):16-22

11 Cuéllar TA, Riatiga ID, Romero WG, Aponte VH. Patrón sensibilidad/resistencia de bacterias según los urocultivos de pacientes con IVU en el Hospital de San José. Revista Urologia Colombiana. 2011;20(02):25-32

12 Pfeifer Y, Cullik A, Witte W. Resistance to cephalosporins and carbapenems in Gram-negative bacterial pathogens. Int J Med Microbiol 2010;300(06):371-379

13 Grupo Para el Control de la Resistencia Antimicrobiana en Bogotá (GREBO) [homepage en Internet] Bogota: Secretaria Distrital de salud de Bogota; 2016http://www.grebo.org

14 Palacio RCTP, Dell'Aqua G, Varela L, Hortal G. M. Resistencia a los antibióticos de patógenos bacterianos aislados de infecciones sistémicas: estudio cooperativo. Rev Med Urug (Montev) 1998; 14:120-133

15 Gavazzi G, Delerce E, Cambau E, et al. Diagnostic criteria for urinary tract infection in hospitalized elderly patients over 75 years of age: a multicenter cross-sectional study. Med Mal Infect 2013;43(05):189-194

16 Gordon LB, Waxman MJ, Ragsdale L, Mermel LA. Overtreatment of presumed urinary tract infection in older women presenting to the emergency department. J Am Geriatr Soc 2013;61(05):788-792

17 Chávez-Valencia V, Gallegos-Nava S, Arce-Salinas CA. Patrones de resistencia antimicrobiana y etiología en infecciones urinarias no complicadas. Gac Med Mex 2010;146(04):269-273

18 Machado-Alba JE, Murillo-Muñoz MM. Evaluación de sensibilidad antibiótica en urocultivos de pacientes en primer nivel de atención en salud de Pereira. Rev Salud Publica (Bogota) 2012;14(04):710-719

19 Gómez ECP, Plata SM, Sejnaui J. Resistencia de la E.coli en urocultivos de pacientes con sospecha de infección urinaria intra y extrahospitalaria en la Fundación Santa Fe de Bogotá. Revista Urologia Colombiana 2009;18(01):53-58 